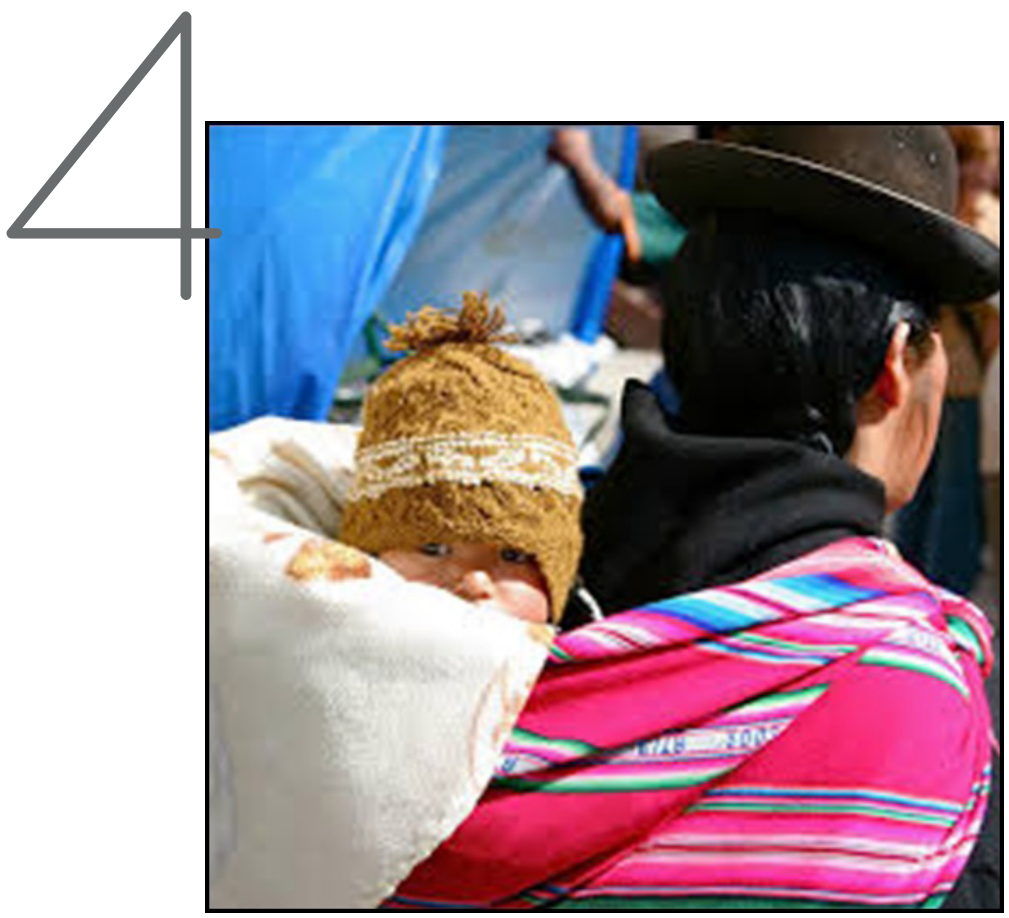

www.google.com.ec/search?q=ni\%C3\%B1os+cargados+en+la+espald a\&tbm=isch\&imgil=wuefWmPnVTZqbM\%253A\%253Bhttps $\% 253 \mathrm{~A} \% 25$ 2F\%252Fencrypted-

\title{
LA LENGUA EN EL PATRIMONIO
}

\author{
Encalada Vásquez, Oswaldo
}

Correspondencia: osencava@uazuay.edu.ec 


\section{Resumen}

El presente acercamiento a la lengua popular -sobre todo en el ámbito andino del Ecuador- intenta centrarse en dos áreas específicas de la expresión:

- Los mecanismos para la construcción de los apreciativos (aumentativos, diminutivos y despectivos) y los superlativos.

- Un análisis somero del léxico referente a la anatomía, con lo que se desea poner de manifiesto que existen iguales fórmulas retóricas usadas por lenguas diferentes para la designación de las parte del cuerpo.

Palabras clave: Lengua popular, patrimonio lingüístico, apreciativos, superlativos, anatomía popular.

\section{LANGUAGE IN CULTURAL HERITAGE}

\section{Abstract}

The present approach towards popular language - most of all in the Andean area of Ecuador - focuses on two specific areas of expression:

- The mechanisms used for the construction of appreciative morphemes (augmentatives, diminutives and pejoratives) and superlatives.

- A superficial analysis of anatomy lexicon, which presents similar rhetorical formulas used by different languages for the naming of body parts.

Keywords: Popular language, linguistic heritage, appreciative morphemes, superlatives, popular anatomy. 


\section{INTRODUCCIÓN}

Dentro de las múltiples posibilidades que nuestra lengua mestiza ofrece para el estudio de los valores patrimoniales, optaremos, en esta ocasión, por dos campos:

1. La construcción de los apreciativos y los superlativos.

2. Una visión sobre el léxico de la anatomía popular.

\section{LA CONSTRUCCIÓN DE LOS APRECIATIVOS Y LOS SUPERLATIVOS}

-Los apreciativos son las formas sustantivales que permiten designar lo pequeño (diminutivo) o aquello que se aprecia; lo grande (aumentativo) y aquello que se desprecia (despectivo).

Hace casi 2500 años Platón (427-347) A.C.) en uno de sus diálogos -el Cratilo o del lenguaje- dice: "El hombre es la medida de todas las cosas". (1979: 251). Esto significa, para nosotros, que lo humano puede ser proyectado al mundo, y mediante el contraste se puede crear conocimiento.

En quichua los apreciativos son verdaderamente comparativos originados e irradiados de la órbita familiar o, por lo menos, del cuerpo humano.

En el año 1600, aproximadamente, el inca Garcilaso de la Vega manifiesta lo siguiente:

"Cerca del cerro Potocchi hay otro cerro pequeño, de la misma forma que el grande, a quien los indios llaman Huayna Potocchi, que quiere decir Potocchi el 
Mozo, a diferencia del otro grande, al cual, después que hallaron el pequeño, llamaron Hatun Potocsi o Potocchi, que todo es uno, y dijeron que eran padre y hijo". (Garcilaso II, 1776:206).

Como se puede ver, lo pequeño es huayna (mozo o joven), en comparación con un adulto, que es grande (hatun).

En la edición de 2008 de la Historia natural y moral de las Indias de Josef de Acosta, el crítico Fermín del Pino Díaz, al mencionar lo del Huayna Potocchi de Garcilaso, comenta: "Del mismo modo que se Ilama Huayna Picchu a un cerro más pequeño situado al lado del famoso Machu Picchu". (Acosta, 2008: 147). Aquí la oposición se establece entre huayna y machu, que significa viejo, anciano; mientras que Picchu significa cima, cumbre, cresta.

Para el año 1901 Lobato publica su Arte y diccionario quechua-español. En él, al referirse a los dedos de la manos se encuentra lo siguiente: mama rucana (pulgar) y sullka rucana (el meñique). Aquí tenemos un aumentativo (mama rucana significa literalmente dedo madre) y un diminutivo (sullka rucana, literalmente dedo menor de edad, el hermano menor de los dedos, que eso es lo que significa sullka).

Ya en el caso de las formas populares del español ecuatoriano tenemos que hay algunas opciones para formar aumentativos o diminutivos. La opción para lo pequeño es usar el sustantivo huahua, traducido como niño - niña; pero, ya con valor diminutivo se traduce como sufijo de diminutivo o como pequeño. Así tenemos, en quichua, 
huahua rumi (piedrita, piedra pequeña), huahua tanda (pancito, panecillo, pan pequeñito). Y ya en el español mestizo y popular encontramos formas como huahua piedra, en oposición a la mama piedra. En este caso se trata de un molino manual formado por dos piedras. La mama piedra (la grande y la que no se mueve) y la huahua piedra (la pequeña y la que gira).

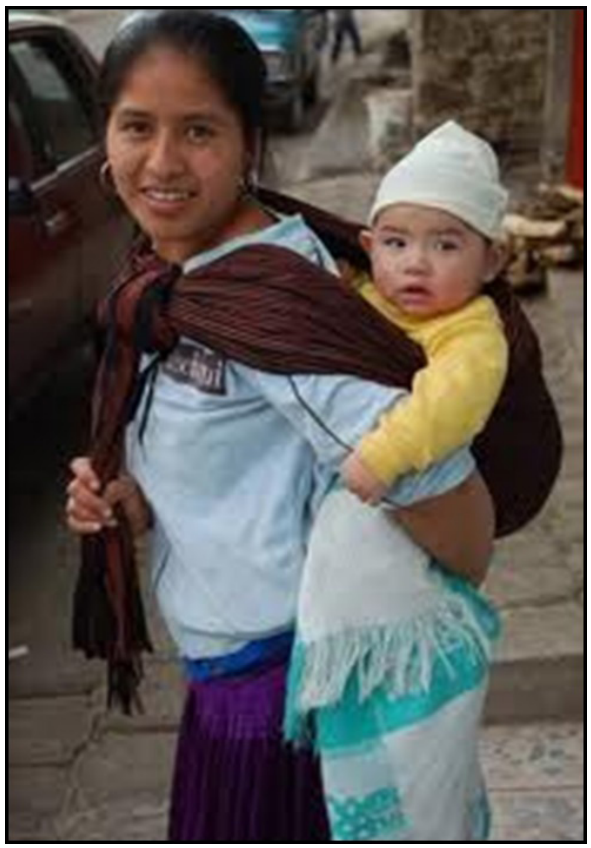

Gráfico 1. Una madre -mama- (lo grande) cargada a su hijo huahua- (lo pequeño).

www.google.com.ec/search?q=mujeres+indigenas+cargadas+con+niños

Otros ejemplos son: huahua cuchara (cuchara pequeña, cucharilla) frente a la cuchara mama, objeto que no se puede decir que sea un cucharón. La cuchara mama 
es de madera y tiene realmente la forma de una cuchara mientras que el cucharón es mucho más cóncavo. Sin embargo en el léxico español sí existe el equivalente de la cuchara mama (llamada también como mama cuchara), es la hataca, definida en el DRAE como: "Cierto cucharón o cuchara grande de palo". (DRAE, 2001: 1191).

Otra pareja de diminutivo - aumentativo es la formada por el mama dedo y el huahua dedo (generalmente el dedo gordo del pie, y el más pequeño). La palabra huahua puede apocoparse, y en este caso va pospuesta, como si fuera un auténtico sufijo: Tandahua = pancito, panecillo; huasihua $=$ casita .

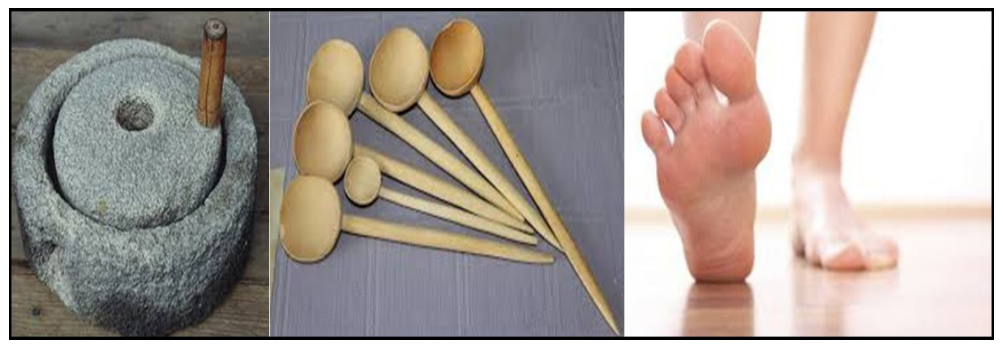

Gráfico 2. La comparación entre mama y huahua. La mama piedra y la huahua piedra; una huahua cuchara en medio de cucharas mamas; el mama dedo y el huahua dedo. (/www.google.com.ec/ search?q=molinos+de+piedra+antiguos), (www.google.com.ec/ search?q=cuchara+de+palo), (imagenes+del+pie\&tbm=isch).

En una variación de esta fórmula se puede usar la palabra rucu (para lo grande, como usaba el quichua machu). En este caso el aumentativo se vuelve ya declaradamente despectivo. Por ejemplo: Huarmirucu (mujerón, literalmente mujer vieja), umarucu (cabezón, literalmente cabeza de viejo o cabeza vieja), allcurucu ((perrote, perrazo, literalmente perro viejo) Jatunrucu (enorme, literalmente gran viejo). 
Respecto de huahua y rucu, en la geografía ecuatoriana tenemos una pareja de picos montañosos: el Huahua Pichincha (escrito generalmente como Guagua, en el norte) y el Rucu Pichincha. El primero tiene 4794 metros de altura, mientras que el segundo, el Rucu Pichincha tiene 4698 m. Lo que significa que la oposición, en este caso, no se refiere realmente al tamaño sino a la actividad volcánica. El Huahua Pichincha es volcán todavía activo; el otro, no.

La designación mediante un componente léxico designador de la infancia ocurre también en otras lenguas, como es el caso del inglés, donde, para formar diminutivos se usa la palabra baby = niño. Así: baby brother (hermanito), baby sister (hermanita), baby carriage (cochecito para niños pequeños).

Otra forma de hacer aumentativos es mediante la palabra quichua sapa, que significa lleno de, abundante en, solo... Así: umasapa (cabezón, literalmente lleno de cabeza o solo cabeza), maquisapa (bracilargo, literalmente lleno de o solo brazos. El maquisapa es un monito del oriente ecuatoriano, que se caracteriza por tener los brazos largos).

Hay otra manera de construir esta especie de comparativos (aumentativos y diminutivos) y es con la adición de las marcas de género, como es el caso de cari y huarmi (para lo que tiene mayor o menor tamaño). Así, Caricocha (laguna grande, literalmente laguna macho, varón) y Huarmicocha (laguna pequeña, literalmente laguna mujer o hembra).

Hace unos pocos años en nuestra Artrología (2008) habíamos planteado ya este tema:

Así también el mayor o menor tamaño se asigna al mundo masculino $o$ al femenino. Todo esto tiene una evidente base física, según los antropólogos: "por término medio los hombres miden 11.6 centímetros más que las mujeres". (Harris, 2002:257). Esto quiere decir que si se concibiera a los seres 
humanos únicamente como siluetas, los altos serían hombres, y los más pequeños serían mujeres.

Esta diferencia puede trasladarse fuera del mundo humano. Así, en el campo vegetal, si hay dos especies o variedades muy parecidas, la que sea de menor tamaño será la hembra:

"Chaquino macho en la provincia de Cuenca, y estoraque en la de Mainas, es el que da uno de los mejores bálsamos llamados del Perú (...) chaquino hembra, árbol poco menor con la hoja como el durazno.

Estoraque macho. Es árbol grande de hojas como de hiedra (...) Estoraque hembra, árbol más femenino de la especie precedente, con la diferencia de dar un fruto más pequeño, redondo". (Velasco, 1961:97-98).

La diferencia de tamaño entre los vegetales aparece también en España:

Se habla del abrótano macho: "Planta herbácea de la familia de las compuestas, de cerca de un metro de altura": Es decir, de 10 decímetros. Abrótano hembra: "planta herbácea de la familia de las compuestas, de cuatro a seis decímetros de altura". (DRAE, 2001:13)

La diferencia de tamaño puede trasladarse al mundo inanimado. Así, de dos cerros cercanos, el más alto será el macho. Se habla de dos montañas ecuatorianas: "Se trata del Carihuairazo, el compañero del Chimborazo que está tan cerca de este que los indígenas lo llaman Chimborazo hembra". (Hassaurek, 1997:88).

En el campo específico de los despectivos, el quichua usa las palabras siqui y sapa. Respecto de siqui (que proviene del campo de la anatomía humana, y que significa trasero) hay que señalar que sirve para - a partir de sustantivos o verbos- crear adjetivos despectivos. Así: 
measiqui, el que acostumbra a orinarse en la ropa. Es adjetivo que se aplica generalmente a los niños pequeños (literalmente se entiende: trasero que mea), puñuisiqui, dormilón (literalmente trasero que duerme), huacaysiqui, llorón (literalmente trasero que Ilora), huatasiqui, panzón, barrigón, (literalmente trasero de barriga).

Con sapa, en función de despectivo, se pueden ver los mismos ejemplos ya mencionados arriba: umasapa, y agregamos casos como rucusapa (viejote), chaquisapa (patudo).

Los superlativos. En quichua los superlativos pueden formarse con la posposición de palabras como supay (diablo) o sapa. Por ejemplo: Ashcasupay (acchca = mucho), bastantísimo; juchasapa = pecadorazo; Ilullasapa = mentirosísimo, shimisapa = habladorazo, muy indiscreto (literalmente solo boca).

Y por último, se pueden crear superlativos con la adición de palabras como monstruo (pronunciado como monstro - monstrro); en casos como monstro caro (carísimo), monstro feo (feísimo). O se puede agregar una especie de adverbio, que es juin, palabra al parecer quichua y que significa muy, mucho. Así: juin duro (durísimo), juin amargo (amarguísimo).

\section{EL LÉXICO DE LA ANATOMÍA POPULAR}

Entre ciertas capas de hablantes, y también en ciertos contextos lingüísticos se pueden escuchar palabras que podrían ser catalogadas como de carácter popular, frente a otras que, más o menos con el mismo significado, 
suelen ser usadas en medios más cuidadosos o de, supuestamente, mayor cultura. Es el caso, por ejemplo, de los siguientes términos:

\begin{tabular}{|l|l|}
\hline \multicolumn{1}{|c|}{ Forma popular } & \multicolumn{1}{|c|}{ Forma más "culta" } \\
\hline $\begin{array}{l}\text {-Cogote (parte superior y posterior el } \\
\text { cuello. Su etimología es cocote, que } \\
\text { viene de coca = cabeza). }\end{array}$ & $\begin{array}{l}\text {-cuello (forma menos } \\
\text { precisa). }\end{array}$ \\
\hline $\begin{array}{l}\text {-Pescuezo (Su significado es similar al } \\
\text { de cogote. En cuanto a la etimología, } \\
\text { viene de post = después; y cogote) }\end{array}$ & -cuello \\
\hline $\begin{array}{l}\text {-Gañote (garganta. Viene de cañón, } \\
\text { en el sentido de tubo). }\end{array}$ & $\begin{array}{l}\text {-garganta (su etimología es } \\
\text { la onomatopeya garg). }\end{array}$ \\
\hline $\begin{array}{l}\text {-Gargüero (garganta. Su etimología } \\
\text { es garg). }\end{array}$ & -garganta \\
\hline $\begin{array}{l}\text {-Gaznate (garganta. Su etimología es } \\
\text { incierta). }\end{array}$ & -garganta \\
\hline $\begin{array}{l}\text {-Panza (viene del latín pantex, con el } \\
\text { mismo significado) }\end{array}$ & -vientre \\
\hline $\begin{array}{l}\text {-Huata (del quichua, significa barriga, } \\
\text { isla, animal doméstico) }\end{array}$ & -vientre \\
\hline -Barriga (derivado de barrica, tonel) & -vientre \\
\hline -Canilla (derivado diminutivo de caña) & -tibia \\
\hline $\begin{array}{l}\text {-Rabadilla (derivación de rabada, y } \\
\text { esta, de rabo) }\end{array}$ & $\begin{array}{l}\text {-cadera (su sentido original } \\
\text { es silla, cátedra). }\end{array}$ \\
\hline $\begin{array}{l}\text {-Espinazo (derivado aumentativo de } \\
\text { espina) }\end{array}$ & -espina dorsal \\
\hline $\begin{array}{l}\text {-Paleta (derivado de pala) } \\
\text {-Pupo (proviene del quichua, significa: } \\
\text { ombligo, protuberancia de ciertos } \\
\text { frutos) }\end{array}$ & $\begin{array}{l}\text {-omóplato (su etimología } \\
\text { significa: parte plana de la }\end{array}$ \\
\hline
\end{tabular}

Estas formas de lengua han sido incorporadas a las expresiones estéticas y de la sabiduría popular, como es 
el caso de los refranes y de las coplas. He aquí algunos casos:

Refranes: - Cuando un perro se traga un hueso confianza tiene en su pescuezo.

- Gargüero que no agradece ser de perro parece.

\section{Coplas:}

Ojitos de indio borracho, Nariz de pupo de lima, Boca de bolsa rasgada, ¡Bonita es mi carishina! (Mera II, s/fecha: 162).

Las mujeres de El Pangui No saben ni dar un beso En cambio las de Chinchipe Te comen hasta el pescuezo. (Márquez et al. 1995:70).

Las formas metafóricas de la designación están presentes en todas las lenguas. $Y$ esto ocurre, naturalmente, también con el quichua, con el mismo valor retórico y con la misma eficacia que en otras lenguas. Por ejemplo:

- Tibia (significa flauta). El quichua dice: chaquipingullo (literalmente: pingullo de la pierna)

- Empeine (designado así por cierta semejanza de la parte anatómica del pie con un peine). El quichua dice: chaquipata (literalmente: meseta del pie).

- Palma. El quichua dice: maquipampa, palma de la mano (literalmente llano de la mano). 


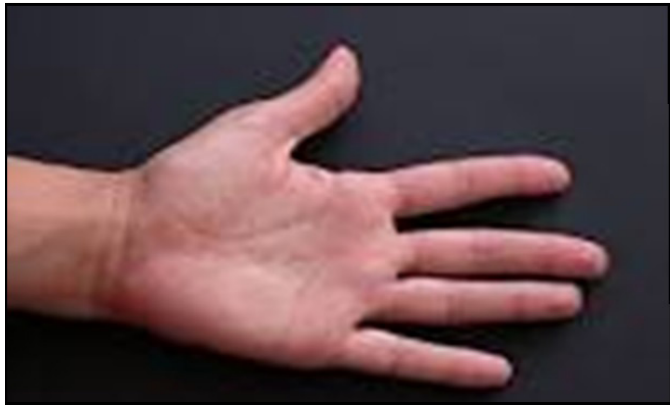

Gráfico 3. La palma de la mano o maquipampa, en quichua. www.google.com.ec/\#q=palma+dela+mano

- Clavícula (su significado real es llavecita, puesto que es un derivado diminutivo de clavis, que es llave, en latín. La explicación se basa en una metáfora entre la forma de este hueso y una llave). El quichua dice: pilischaca (literalmente puente -chaca- de los piojos).

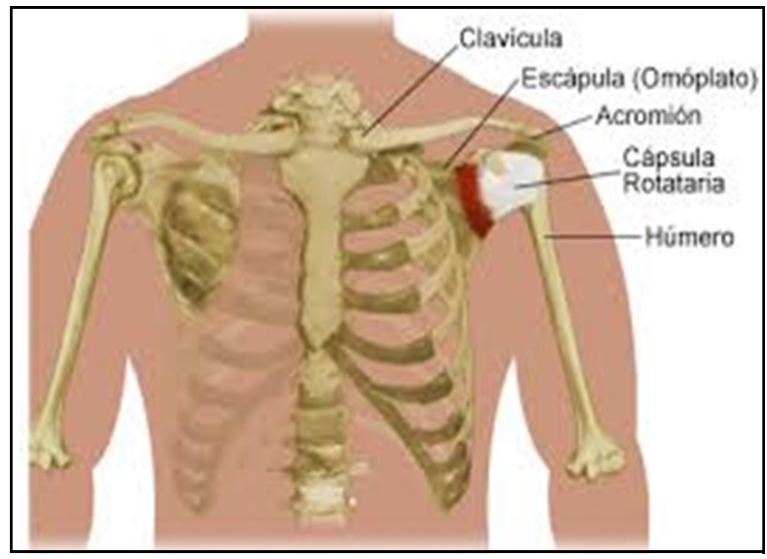

Gráfico 4. La clavícula (llavecita) y el pilischaca (puente de los piojos). www.google.com.ec/search?q=clavicula 
- Cóccix o coxis (es palabra griega y significa cuclillo. La relación significativa con esta ave europea cuyo canto suena algo así como cucú, es opaca para este momento). El cóccix, en términos anatómicos, es lo que queda de una antigua cola vestigial. El quichua dice: chupatullu (traducido como hueso -tullu- del rabo).

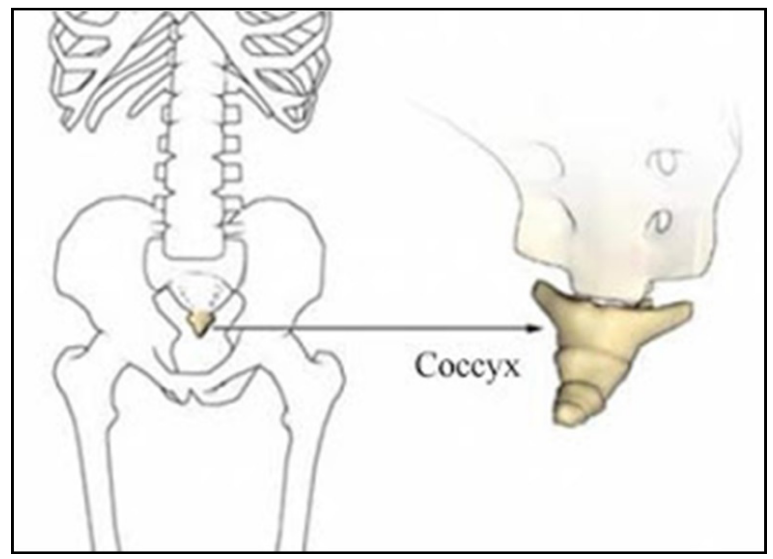

Gráfico 5. El cóccix -coxis - y el chupatullo, el hueso del rabo, en quichua.

www.google.com.ec/search?q=coxis

- Pantorrilla (derivado del latín pantex = barriga grande, panza. La razón es visible puesto que esta parte parece realmente una especie de vientre pegado a la pierna). EI quichua dice: chaquichichun (literalmente pierna preñada o embarazo de la pierna). En griego clásico la pantorrilla tiene una designación igualmente llamativa. Es gastrokreme, lo que se puede traducir como barriga colgada. 


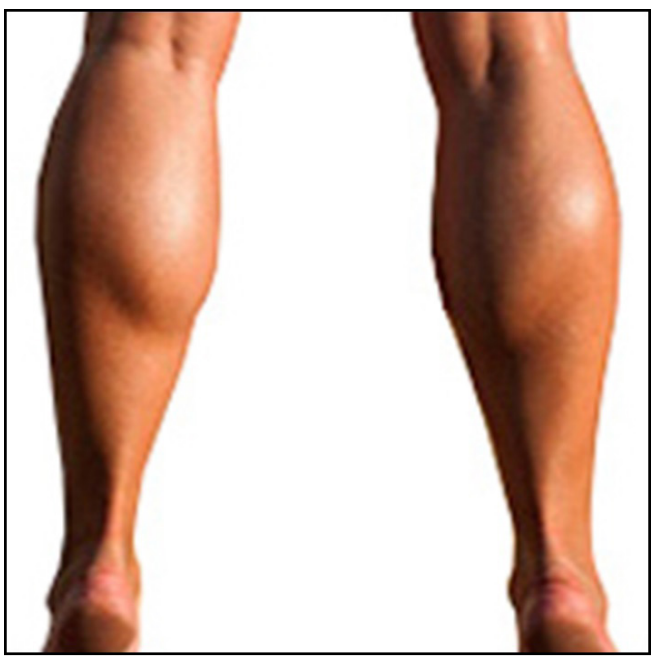

Gráfico 6. La forma de la pantorrilla recuerda claramente la imagen de una barriga. El quichua dice chaquichichun -barriga preñada- y el griego, gastrokreme - barriga colgada.

www.google.com.ec/search?q=pantorrillas+gordas

-Ombligo (derivado diminutivo del latín umbo, que significa centro abultado del escudo, saliente). El quichua dice: pupu (traducido como protuberancia de ciertas frutas, como la lima). 


\section{BIBLIOGRAFÍA}

Acosta, Joseph de, 2008 Historia natural y moral de las Indias, Madrid, Consejo Superior de Investigaciones Científicas.

Encalada Vásquez, Oswaldo, 2008 Artrología, Quito, UDA/CONESUP.

Garcilaso de la Vega, 1976 Comentarios reales II, Caracas, Biblioteca Ayacucho.

Harris, Marvin, 2002 Nuestra especie, Barcelona, Alianza Editorial.

Hassaurek, Friedrich, 1997 Cuatro años entre los ecuatorianos, Quito, Abya Yala.

Lobato, Juan G.N., 1901 Arte y diccionario quechua - español, Lima, Imprenta del Estado.

Márquez, Luis Guillermo, et al., 1995 Tradiciones de mi pueblo, Loja, Casa de la Cultura Ecuatoriana.

Mera, Juan León,

s/ fecha Cantares del pueblo ecuatoriano II. Guayaquil Quito, Clásicos Ariel.

Platón,

1979 Diálogos, Cratilo, México, Porrúa.

Real Academia Española, 2001 Diccionario de la lengua española, Madrid, Espasa Calpe. 
Velasco, Juan de,

1961 Historia natural, Puebla, Cajica.

www.google.com.ec/search?q=ni\%C3\%B1os+cargados+en+la+ espalda\&tbm=isch\&imgil=wuefWmPnVTZqbM\%253A\%253Bhtt ps\%253A\%252F\%252Fencrypted-

wikipedia.org/wiki/Coxis (Consulta el 7 de marzo de 2014).

www.google.com.ec/search?q=mujeres + indigenas + cargadas $+c$ on+niños

http://lema.rae.es/drae/?val=hataca

www.google.com.ec/search?q=molinos $+d e+$ piedra+antiguos

www.google.com.ec/search?q=cuchara+de+palo), imagenes+de $1+$ pie\&tbm=isch

www.google.com.ec/\#q=palma+dela+mano

www.google.com.ec/search?q=clavicula

www.google.com.ec/search?q=coxis

www.google.com.ec/search?q=pantorrillas+gordas

(El resto de consultas fueron realizadas el 30 de abril de 2014) 